\title{
Significance of an index of insulin resistance on admission in non-diabetic patients with acute coronary syndromes
}

\author{
P J Stubbs, J Alaghband-Zadeh, J F Laycock, P O Collinson, G D Carter, M I M Noble
}

Department of

Cardiology, Northwick Park Hospital, Watford Road, Harrow,

Middlesex HA1 3UJ, UK

P J Stubbs

National Heart and

Lung Institute, Imperial College School of Medicine, Charing Cross Campus, Fulham Palace Road, London W6 8RF, UK M I M Noble

Division of Diagnostic and Investigative

Science, Imperial College School of Medicine, Charing Cross Campus, Fulham Palace Road, London W6 8RF, UK

J Alaghband-Zadeh

G D Carter

Division of

Neurosciences, Imperial College

School of Medicine, Charing Cross

Campus

J F Laycock

Department of Chemical Pathology, Mayday University

Hospital, Croydon,

Surrey, UK

P O Collinson

Correspondence to: Dr Stubbs.

Accepted for publication 5 May 1999

\begin{abstract}
Background-Insulin resistance is associated with ischaemic heart disease and has been proposed as a risk factor for subsequent myocardial infarction.

Aim-To investigate the potential use of a recently proposed insulin resistance index in identifying insulin resistance in patients admitted with an acute coronary syndrome.

Methods-Single centre study of 441 nondiabetic patients admitted with chest pain to a coronary care unit and followed prospectively for a median of three years for outcome. Admission glucose and insulin concentrations were measured and from these values an admission index of insulin resistance (AIRI) calculated. Its association with other known factors in the insulin resistance syndrome, and subsequent outcome, was examined.

Results-The AIRI was greater in patients with myocardial infarction than in a control group without myocardial infarction $(p<0.0001)$. A Cox regression model for subsequent cardiac death identified previous myocardial infarction $(p<0.0001)$, infarct size $(p<0.0001)$, and AIRI ( $p=0.0033)$ as positive risk predictors. Patients of Indian subcontinent ethnic origin had greater AIRI values than white patients: mean (SD) $7.5(1.3) v 4.6$ $(0.2), \mathrm{p}<0.001$.

Conclusions-A simple index of insulin resistance measured on patients admitted with myocardial infarction provides an important predictive measure of poor outcome and is superior to admission glucose measurement. It may be useful in identifying patients admitted with myocardial infarction who could benefit from alternative early management strategies. (Heart 1999;82:443-447)
\end{abstract}

Keywords: myocardial infarction; unstable angina; insulin resistance; glucose

Insulin resistance is defined as impaired sensitivity to the effects of insulin on whole body glucose utilisation. ${ }^{1}$ Its presence is associated with a clustering of cardiovascular risk factors including hypertension, raised plasma very low density lipoprotein (VLDL) triglycerides, and low plasma high density lipoprotein (HDL) cholesterol. $^{2-4}$ Its precise measurement depends on the use of the euglycaemic insulin clamp and appears mainly to affect the non-oxidative (glycogen synthesis) or oxidative pathways of glucose metabolism in skeletal muscle. ${ }^{1}$ The euglycaemic clamp method, however, is not suitable as a screening method for large scale epidemiological studies and so insulin resistance has been inferred by measuring fasting insulin and glucose concentrations. $^{34}$

The results of these studies have shown that the syndrome of insulin resistance appears to be a significant risk factor for coronary events, particularly among patients of south east Asian origin. ${ }^{3}$ Whether it is possible to identify insulin resistant patients on presentation with an acute coronary syndrome has not been examined, and would require simple methodology. It is well known that the stress of an acute coronary syndrome may reveal diabetes for the first time, and admission hyperglycaemia has been shown to be associated with a poor outcome. ${ }^{5}$ One possible method would be to determine the glucose to insulin ratio on admission. This method may be misleading, however, as if both glucose and insulin concentrations are increased the ratio may remain in the "normal" range. Another simple index of insulin resistance - related to the product of the fasting plasma insulin and glucose concentrations divided by the product of a "normalised" insulin (5 $\mathrm{mU} / \mathrm{l})$ and glucose (5 $\mathrm{mmol} / \mathrm{l})$ concentration-has been proposed and recently validated: the fasting insulin resistance index (FIRI). ${ }^{67}$

A similar measure of insulin resistance, based on admission blood samples from non-diabetic patients admitted to hospital with chest pain considered to be of ischaemic origin (an admission insulin resistance index, AIRI), could be of value if it was established that it correlated with known indices of the insulin resistance syndrome, and if such an index were shown to be a predictor of adverse outcome, despite the confounding effect of the physiological hormonal response to stress. ${ }^{8}$ These were our objectives in the present study.

\section{Methods}

This was a single centre study of patients admitted with chest pain to a hospital coronary care unit. Management decisions were based on clinical, ECG, and routine biochemical marker results. Patients admitted to the coronary care unit underwent daily sampling for three days according to the routine cardiac enzyme protocol in the hospital, and the daily creatine kinase, aspartate transaminase, and hydroxybutyrate dehydrogenase estimations 
were used for the final World Health Organisation (WHO) biochemical diagnostic coding of the admissions. ${ }^{9}$ Patients also underwent multiple timed sampling within their admission period for research purposes, approved by the local ethics committee, which included samples taken on admission to the coronary care unit before the initiation of antithrombotic treatment. These coded samples were centrifuged and the serum immediately frozen in liquid nitrogen before storage at $-80^{\circ} \mathrm{C}$. Samples for admission glucose estimation were taken into Vacutainer fluoride oxalate tubes and measured prospectively.

CLINICAL DATA

Full clinical details were recorded on all patients by proforma. Particular attention was paid to previous cardiac history, classification of chest pain before entry, admission clinical findings, inpatient clinical course and management, discharge drug management, and subsequent investigations and treatment. Myocardial infarction was diagnosed using WHO criteria: chest pain, ECG changes, and the finding of cardiac enzyme concentrations at least twice the upper reference limit within the first 48 hours after admission.

Follow up for survival, interventions, and mortality was by examination of hospital records, necropsy results when available, death certificates, general practitioner questionnaire, patient or next of kin questionnaire, with follow up telephone contact if required. Survival status and cause of death were established for all patients. Cause of death was classified according to American Heart Association criteria. ${ }^{10}$ In order to confine the study to nondiabetic patients, all those who were known to suffer from diabetes or who had an admission blood glucose concentration greater than 11 $\mathrm{mmol} / 1$ were excluded from the analysis. ${ }^{511}$

ANALYTICAL METHODS

Aspartate transaminase and hydroxybutyrate dehydrogenase were measured at $37^{\circ} \mathrm{C}$ by optimised methods on a Perspective analyser (American Monitor, Burgess Hill, West Sussex, UK), using the manufacturer's recommended procedures. The manufacturer's reagents were used for aspartate transaminase (reference interval 11 to $55 \mathrm{u} / 1$ ) and commercially supplied reagents used for hydroxybutyrate dehydrogenase (reference interval 90 to 180 $\mathrm{u} / \mathrm{l}$ ) (Merckotest $\mathrm{HBDH}$; BDH Diagnostics, Poole, Dorset, UK).

Total creatine kinase was measured at $30^{\circ} \mathrm{C}$ by optimised methods, using commercially supplied reagents (CK NAC opt; BCL, Lewes, Sussex, UK; reference interval $<120 \mathrm{u} / \mathrm{l}$ ). All determinations were made using an RA 1000 analyser (Bayer Technicon, Basingstoke, UK).

Troponin $\mathrm{T}$ was determined by an enzyme linked immunosorbent assay (ELISA) using an ES-300 immunoassay analyser (Boehringer Mannheim, Lewes, Sussex, UK), as previously described. ${ }^{12}$ The detection limit for the assay is $0.05 \mathrm{ng} / \mathrm{ml}$. Interassay imprecision values, estimated by coefficient of variation (CV), were $12.3 \%, 7.7 \%$, and $4.2 \%$ at mean troponin $\mathrm{T}$ concentrations of $0.13 \mathrm{ng} / \mathrm{ml}, 1.6 \mathrm{ng} / \mathrm{ml}$, and $7.1 \mathrm{ng} / \mathrm{ml}$, respectively. Intra-assay imprecision values were $2.0 \%$ and $1.9 \%$ at mean troponin $\mathrm{T}$ concentrations of $1.7 \mathrm{ng} / \mathrm{ml}$ and $7.0 \mathrm{ng} / \mathrm{ml}$, respectively. This cardiac specific marker is at its most efficient for diagnosing myocardial damage when measured between 12 and 24 hours after admission and the concentration in this period was used as a measure of infarct size. ${ }^{12}$

Admission cholesterol concentration (reference interval 3.5 to $6.5 \mathrm{mmol} / \mathrm{l}$ ) was measured by a cholesterol oxidase method on a Technicon Axon (Bayer Technicon) by the manufacturer's recommended procedure (within run CV 2.2\%, between run CV 5\%).

Admission triglyceride concentrations (reference interval 0.9 to $2.0 \mathrm{mmol} / \mathrm{l}$ ) were measured by a lipoprotein lipase/glycerol kinase/glycerol phosphate oxidase reaction on a Technicon Axon by the manufacturer's recommended method (within run $\mathrm{CV} 3.0 \%$, between run $\mathrm{CV} \mathrm{5 \% ).}$

Insulin was measured using an ELISA one step sandwich assay which has a $40 \%$ cross reactivity with proinsulin. Its between assay coefficient of variation was $8.2 \%$. Glucose was measured by an automated glucose oxidase method. The AIRI for each blood sample was determined using the admission blood glucose and insulin concentrations divided by the product of "normalised" glucose $(5 \mathrm{mmol} / \mathrm{l})$ and insulin ( $5 \mathrm{mU} / \mathrm{l})$.

STATISTICAL ANALYSES

Baseline demographic variables were expressed as percentages, mean (SEM), or median (range) as appropriate. Comparisons between cohorts were made using Mann-Whitney U tests for continuous variables and $\chi^{2}$ analysis for categorical variables. Correlations were tested by the Rank-Spearman correlation coefficient $\left(r_{\mathrm{s}}\right)$. Multiple regression for survival was examined using the Cox regression statistical model. Forward conditional stepwise regression models were used to rank the relative importance of variables as risk predictors for outcome. For all statistical evaluations, a two tailed $p$ value of 0.05 or less was considered to be statistically significant. The end point studied was cardiac death as first event. Non-cardiac deaths were treated as censored observations in the statistical analyses.

\section{Results}

Admission glucose and insulin concentrations were available for 441 patients admitted with chest pain who were non-diabetic and had an admission glucose concentration $<11 \mathrm{mmol} / 1$. $^{5}$ By the criteria defined above, the index admission was retrospectively classified into two cohorts, one with myocardial infarction (infarct group; 195 patients) and one without myocardial infarction (non-infarct group; 246 patients). These cohorts were followed for a median of 1053 days (lower quartile 875, upper quartile 1342). The baseline demographic variables for these cohorts are shown in table 1. Overall, $18.5 \%$ (36/195) of the infarct 
Table 1 Descriptive data of the two groups of patients in the study: patients with myocardial infarction (MI) and without myocardial infarction (non-MI)

\begin{tabular}{llcc}
\hline & MI & Non-MI & p Value \\
\hline Number & 195 & 246 & \\
Sex (\% male) & 70 & 66 & $0.60^{\star}$ \\
Age (years) & $61.4(0.8)$ & $57.9(0.8)$ & 0.003 \\
Previous MI (\%) & 18 & 32 & $0.001^{\star}$ \\
Hypertensive (\%) & 26.0 & 24.5 & $0.79^{\star}$ \\
Asian origin (\%) & 18 & 22 & $0.29^{\star}$ \\
Time (h) from initial worst pain to CCU & & & \\
$\quad$ (median (range)) & $4.6(3$ to 8$)$ & $5.0(3$ to 9$)$ & 0.23 \\
Admission Killip score & $9.6(0.2)$ & $8.1(0.2)$ & $<0.0001$ \\
Admission plasma glucose (mmol/l) & $7.6(0.1)$ & $6.2(0.1)$ & $<0.001$ \\
Admission plasma insulin (mU/l) & $16.5(0.9)$ & $16.6(1.3)$ & 0.10 \\
AIRI & $5.1(0.3)$ & $4.6(0.5)$ & $<0.0001$ \\
Admission plasma cholesterol (mmol/l) & $6.4(0.1)$ & $6.2(0.15)$ & 0.15 \\
Admission plasma triglycerides (mmol/l) & $1.6(0.1)$ & $1.7(0.1)$ & 0.29 \\
Mody mass index (kg/m ${ }^{2}$ ) & $25.2(0.3)$ & $25.9(0.4)$ & 0.28 \\
Waist/hip ratio & $0.96(0.01)$ & $0.96(0.01)$ & 0.79 \\
Revascularisation (\%) & 28 & 20 & $0.06^{\star}$ \\
\hline
\end{tabular}

Values are mean (SEM) unless stated otherwise.

$\mathrm{p}$ Values are given for comparisons between the two groups using the Mann-Whitney $\mathrm{U}$ test or $\chi^{2}$ analysis ${ }^{\star}$.

AIRI, admission insulin resistance index; CCU, coronary care unit.

Table 2 Spearman correlation coefficients $(r)$ and their $p$ values relating admission insulin resistance index (AIRI) and various other measurments on admission in patients with myocardial infarction (MI) and those without infarction (non-MI)

\begin{tabular}{lrccccc}
\hline & \multicolumn{2}{l}{$M I$} & & \multicolumn{2}{c}{ Non-MI } \\
\cline { 2 - 3 } \cline { 6 - 7 } & $r_{s}$ & $p$ Value & & $r_{s}$ & $p$ Value \\
\hline Age & -0.17 & 0.03 & & 0.07 & 0.3 \\
Body mass index & 0.31 & $<0.0001$ & & 0.39 & $<0.0001$ \\
Waist/hip ratio & 0.23 & 0.060 & & 0.33 & 0.011 \\
Cholesterol & 0.03 & 0.78 & & 0.16 & 0.07 \\
Triglycerides & 0.12 & 0.16 & & 0.32 & $<0.0001$ \\
Diagnostic troponin $\mathrm{T}$ & 0.04 & 0.64 & & 0.07 & 0.39 \\
Killip score & 0.14 & 0.06 & & 0.09 & 0.22 \\
\hline
\end{tabular}

group and $10.6 \%(25 / 246)$ of the non-infarct group died from a cardiac cause $\left(\chi^{2}=6.3\right.$, $\mathrm{p}<0.01)$.

Patients with a final diagnosis of myocardial infarction were significantly older and had higher admission Killip scores and glucose concentrations than the non-infarct group (table 1). Although the admission insulin concentrations were similar in the two groups, the AIRI scores were higher in the infarct group than in the non-infarct group $(\mathrm{p}<0.0001)$. There were no significant differences in body mass indices or waist-hip ratios between the groups. Patients in the non-infarct group had a higher frequency of previous myocardial infarction than the infarct group (32\% v 18\%, $\mathrm{p}=0.001)$. There were highly significant correlations between the admission AIRI score and body mass index in both groups (table 2). In the infarct group there were weak correlations between the AIRI scores, the waist-hip ratios, and the admission Killip scores, and there was a significant negative correlation between AIRI and age. In the non-infarct group there were significant correlations between the AIRI scores, the waist-hip ratios, and the admission triglyceride concentrations, and a weak correlation with the admission cholesterol concentrations (table 2).

REGRESSION ANALYSES

Patients with myocardial infarction

The AIRI values for this group were entered into a one step multivariate Cox regression analysis along with age, previous myocardial infarction, admission Killip score, thrombolysis, infarct size, and subsequent revascularisation for the end point cardiac death. The $\chi^{2}$ results together with the significances and 95\% confidence intervals for risk are shown in table 3. The AIRI was a significant independent risk predictor for this end point $\left(\chi^{2}=8.7\right.$, $\mathrm{p}=0.0033)$ and ranked third behind previous myocardial infarction and infarct size. As expected, thrombolysis was a significant negative predictor of subsequent cardiac death (table 3). In order to rank AIRI as a risk predictor, the four significant variables were entered into a forward conditional stepwise Cox regression model, the results of which are shown in table 4. In this analysis AIRI retained its significance $(\mathrm{p}=0.006)$ behind infarct size and previous myocardial infarction. Admission glucose was entered into these models either as a continuous variable or stratified as $>8$ $\mathrm{mmol} / \mathrm{l}$ and failed to achieve significance ( $\mathrm{p}=0.16$ and 0.13 respectively).

\section{Patients without myocardial infarction}

The AIRI values for this group were entered into a one step multivariate Cox regression analysis along with age, previous myocardial infarction, accelerated angina in the 48 hours before admission, rest pain on admission, admission Killip score, troponin $\mathrm{T}$ concentration at 12-24 hours from admission, and subsequent revascularisation, for the end point cardiac death. The $\chi^{2}$ results together with the significances and $95 \%$ confidence intervals are shown in table 5. The AIRI was not a significant risk predictor for this end point. Admission glucose appeared to be a superior predictor to AIRI in this patient group but did not quite reach conventional significance (table $5)$.

\section{Patients of ethnic origin from the Indian} subcontinent

These Asians formed $18 \%$ of the infarct group (35 of 195) and $22 \%$ of the non-infarct group (54 of 246). They originated almost exclusively

Table 3 One step multivariate Cox regression analysis identifying predictors of subsequent cardiac death in patients with myocardial infarction

\begin{tabular}{|c|c|c|c|}
\hline & p Value & Relative risk & $95 \% C I$ \\
\hline Age & 0.1 & 1.04 & 0.99 to 1.1 \\
\hline AIRI & 0.0033 & 1.13 & 1.0 to 1.2 \\
\hline Infarct size & 0.0004 & 1.06 & 1.0 to 1.1 \\
\hline Killip score & 0.94 & 1.0 & 0.87 to 1.2 \\
\hline Previous MI & 0.0003 & 7.4 & 2.5 to 22.1 \\
\hline \multicolumn{4}{|l|}{ Subsequent } \\
\hline revascularisation & 0.16 & 0.22 & 0.03 to 1.8 \\
\hline Thrombolysis & 0.017 & 0.25 & 0.08 to 0.80 \\
\hline
\end{tabular}

AIRI, admission insulin resistance index; CI, confidence interval; MI, myocardial infarct.

Table 4 Forward conditional stepwise regression analysis ranking significant predictors of subsequent cardiac death in patients with myocardial infarction

\begin{tabular}{lcll}
\hline & $p$ Value & Relative risk & $95 \%$ CI \\
\hline Infarct size & $<0.0001$ & 1.07 & 1.0 to 1.1 \\
Previous MI & $<0.0001$ & 10.3 & 3.4 to 30.5 \\
AIRI & 0.006 & 1.13 & 1.0 to 1.2 \\
Thrombolysis & 0.009 & 0.22 & 0.07 to 0.7
\end{tabular}

AIRI, admission insulin resistance index; CI, confidence interval; MI myocardial infarct. 
Table 5 One step multivariate Cox regression analysis identifying predictors of subsequent cardiac death in patients who did not have myocardial infarction

\begin{tabular}{|c|c|c|c|}
\hline & $p$ Value & Relative risk & $95 \% C I$ \\
\hline Accelerated angina & 0.66 & 0.94 & 0.35 to 5.2 \\
\hline Age & 0.03 & 1.07 & 1.3 to 1.5 \\
\hline AIRI & 0.65 & 0.85 & 0.92 to 1.1 \\
\hline Killip score & 0.72 & 0.95 & 0.71 to 1.3 \\
\hline Previous MI & 0.002 & 23.09 & 2.9 to 20.0 \\
\hline Rest pain & 0.81 & 1.35 & 0.35 to 3.9 \\
\hline $\begin{array}{l}\text { Subsequent } \\
\text { revascularisation }\end{array}$ & 0.22 & 0.28 & 0.03 to 2.4 \\
\hline $\begin{array}{l}\text { Troponin } \mathrm{T} \\
\text { concentration }\end{array}$ & 0.004 & 2.31 & 1.3 to 3.5 \\
\hline Admission glucose & 0.057 & 1.89 & 0.98 to 3.6 \\
\hline
\end{tabular}

AIRI, admission insulin resistance index; CI, confidence interval; MI, myocardial infarct.

from the Punjab region of northern India and were mainly Sikhs. In the infarct group, the average AIRI was significantly higher in the Asian subgroup than in non-Asians $(7.5$ (1.3) $v$ $4.6(0.2)$, respectively; $\left.\chi^{2}=11.8, \mathrm{p}<0.0006\right)$. The study was insufficiently powered to allow separate analysis of prognosis for each ethnic subgroup.

\section{Discussion}

Our study showed that insulin resistance, as determined by the admission insulin resistance index, was a significant predictor of a poor outcome in patients admitted with myocardial infarction and is a superior predictor to the admission glucose measurement. As shown in table 2, the AIRI correlated with various indices of the insulin resistance syndrome despite the effects of the physiological hormonal response to stress; in particular it was higher in the Asian patients studied, for whom insulin resistance appears to be a major risk factor for heart disease. ${ }^{4}$ Repeating the regression analysis in table 4 controlling for Asian patients weakened the significance but AIRI remained an independent predictor $(p=0.017$, relative risk $1.12,95 \%$ confidence interval 1.02 to 1.23 ).

The lack of correlation between AIRI and admission triglyceride concentrations in the myocardial infarction group probably reflected the added effect of the stressor hormones in this group. ${ }^{8}$ AIRI was not associated with an adverse outcome in non-infarct patients although this study confirms the importance of minor myocardial damage, identified by troponin $\mathrm{T}$ presence, as a predictor of cardiac death in this group. ${ }^{13}$

The simple methodology required to measure and calculate an admission insulin resistance index makes it suitable for large scale epidemiological studies. It is derived in the same way as the fasting insulin resistance index (FIRI), which correlates significantly with the homeostasis model assessment (HOMA) estimate of insulin resistance using a computer solved model based on fasting plasma glucose and insulin concentrations. ${ }^{14}$ Similarly, it correlates significantly with the measure of insulin sensitivity provided by the minimal model method of Bergman et al, ${ }^{15}$ and an inverse correlation between the $\log _{10}$ of this measure and a measure of insulin mediated glucose disposal has recently been demonstrated. ${ }^{7}$ However, other workers ${ }^{1617}$ have not concurred with these findings, and this may be accounted for by differences in the methodologies used for insulin measurement. ${ }^{7}$

Having demonstrated the prognostic significance of this admission insulin resistance index, how may its measurement be clinically useful in patients admitted with myocardial infarction?

The preferred substrate for the infarcting myocardium is glucose, and insulin is necessary to increase glucose entry into muscle cells. Euglycaemic clamp studies on skeletal muscle in insulin resistant patients have shown a reduction in glycogen synthesis as a result of resistance to insulin mediated glucose uptake. ${ }^{1}$ It has recently been demonstrated, using positron emission tomography and clamp studies, that cardiac myocytes in patients with coronary disease also have resistance to insulin mediated glucose disposal. ${ }^{18}$ In the context of the infarcting myocardium, therefore, insulin resistant patients may be exposed to double jeopardy: not only may the important intramyocyte glycogen stores be low and subject to rapid depletion, but further glucose delivery to the ischaemic myocyte will be impaired as well, by the resistance to insulin mediated glucose disposal. Support for this hypothesis comes from the recently published diabetes, insulin, and glucose in acute myocardial infarction (DIGAMI) study, which examined the effect of metabolic support using glucose-insulinpotassium (GIK) infusions and subsequent insulin in diabetic patients sustaining a myocardial infarct. ${ }^{19}$

The subgroup which particularly benefited from this regimen in terms of reduction in subsequent cardiac morbidity was the diabetic group not receiving insulin treatment before admission (that is, type 2 diabetes mellitus, most of whom are insulin resistant). There has been a recent resurgence of interest in the concept of metabolic support for ischaemic myocardium ${ }^{8}$ and a proposal that a large study be initiated to retest the value of GIK treatment in the thrombolytic era in patients admitted with myocardial infarction. ${ }^{20}$ Earlier reservations that increased glycolysis in ischaemic tissue may be counterproductive because of an accumulation of harmful protons (for example superoxides) and lactate ${ }^{21}$ may now not be valid, as the routine use of thrombolysis with successful reperfusion of the occluded vessel should allow washout of these metabolites. The currently available data suggest that insulin resistant patients may have the most to gain from this treatment and the use of the simple admission index of insulin resistance proposed in this study may help identify non-diabetic insulin resistant subgroups.

\section{CONCLUSIONS}

Our study shows that a simple index of insulin resistance measured on patients admitted with a myocardial infarct provides an important predictive measure of poor outcome and is superior to admission glucose measurement. It may be useful in identifying patients admitted 
with myocardial infarction who could benefit from alternative early management strategies.

1 DeFronzo RA, Tobin JD, Andres R. The glucose clamp technique: a method for quantifying insulin secretion and technique: a method for quantifying insulin

2 DeFronzo RA, Ferrannini E. Insulin resistance. A multifaceted syndrome responsible for NIDDM, obesity, hypertension, dyslipidemia, and atherosclerotic cardiovascular disease. Diabetes Care 1991;14:173-94.

3 McKeigue PM, Shah B, Marmot MG. Relationship of central obesity and insulin resistance with high diabetes prevalence and cardiovascular risk in South Asians. Lancet 1991; i: $382-6$.

4 Caro JF. Insulin resistance in obese and non-obese man. $\mathcal{F}$ Clin Endocrinol Metab 1991;73:691-5.

5 Oswald GA, Smith CCT, Betteridge DJ, et al. Determinants and importance of stress hyperglycaemia in non-diabetic patients with myocardial infarction. BMF 1986;293:91722 .

6 Duncan $\mathrm{MH}$, Singh BM, Wise PH, et al. A simple measure of insulin resistance. Lancet 1995;346:120-1.

7 Cleland SJ, Petrie JR, Morris AD, et al. FIRI: a fair insulin Cleland SJ, Petrie JR, Morris AD, et al.
resistance index? Lancet 1996;347:770.

8 Oliver MF, Opie LH. Effects of glucose and fatty acids on myocardial ischaemia and arrhythmias. Lancet 1994;343. myocar

9 Working group on the Establishment of Ischaemic Heart Disease Registers. Report of the fifth working group. WHO, Eur 8201 (5). Copenhagen: World Health Organisation, 1971 .

10 Gillum RF, Fortmann SP, Prineas RJ. Internationa diagnostic criteria for acute myocardial infarction and acute stroke. Am Heart f 1984;108:155-8.
11 World Health Organisation. Diabetes mellitus. Report of a WHO Study Group. [WHO Technical Report Series.] Geneva: WHO, 1985:727.

12 Collinson PO, Moseley D, Stubbs P, et al. Troponin T for the differential diagnosis of ischaemic myocardial damage. Ann Clin Biochem 1993;30:11-16.

13 Hamm CW, Ravkilde J, Gerhardt W, et al. The prognostic value of serum troponin $\mathrm{T}$ in unstable angina. $\mathrm{N}$ Englf $\mathrm{Med}$ 1992;327:146-50.

14 Matthews DR, Hosker JP, Rudenski JS, et al. Homeostasis model assessment: insulin resistance and $\beta$-cell function from fasting plasma glucose and insulin concentrations in man. Diabetologia 1985;28:412-19.

15 Bergman RN, Prager R, Volund A, et al. Equivalence of the insulin sensitivity index in man derived by the minimal model method and euglycaemic glucose clamp. Am $7 \mathrm{Clin}$ Invest 1987;79:790-800.

16 Yarnell JWG, Patterson CC, Sweetnam PM. Simple measure of insulin resistance. Lancet 1996;346:1108-9.

17 Del Prato S, Pozzilli P. FIRI: fasting or false insulin resistance index? Lancet 1996;347:132.

18 Reaven GM. Role of insulin resistance in human disease syndrome X): an expanded definition. Annu Rev Med 1993;44:121-31.

19 Malmberg K, Ryden L, Efendic S, et al. Randomized trial of insulin-glucose infusion followed by subcutaneous insulin insulin-glucose infusion followed by subcutaneous insulin tion (DIGAMI study): effects on mortality at 1 year. $₹ \mathrm{Am}$ Coll Cardiol 1995;26:57-65.

20 Opie LH. Glucose and the metabolism of ischaemic myocardium. Lancet 1995;345:1520-21.

21 Neely JR, Grotyohann LW. Role of glycolytic products in damage to ischemic myocardium. Dissociation of adenosine triphosphate levels and recovery of function of reperfused ischemic hearts. Circ Res 1984;55:816-24. 